\title{
Validación del Cuestionario de Motivos de Juego en adolescentes: Diferencias según la gravedad y las actividades de juego
}

\section{Gambling Motives Questionnaire validation in adolescents: Differences based on gambling severity and activities}

\author{
Aris Grande-Gosende*, Víctor Martínez-Loredo*, José Ramón Fernández-Hermida*. \\ *Addictive Behavior Research Group, University of Oviedo.
}

\section{Resumen}

Los motivos de juego han sido considerados como variables clave para comprender el desarrollo de problemas asociados al mismo en adultos. Sin embargo, la literatura sobre las motivaciones de los adolescentes para jugar es escasa. El presente estudio tiene como objetivo explorar la estructura factorial del Cuestionario de Motivos de Juego (GMQ) y analizar las diferencias de los motivos de los adolescentes según la actividad de juego y la gravedad de los problemas asociados. Se evaluó una muestra de 698 jugadores adolescentes $(\mathrm{M}=15,24, \mathrm{DT}=, 76) \mathrm{a}$ los que se les aplicó, además del GMQ, el cuestionario South Oaks Gambling Screen (SOGS-RA). Los jugadores fueron clasificados en tres categorías (estratégicos, no estratégicos, mixtos) según el tipo de juegos utilizados durante el último año. Para el estudio de la estructura interna del GMQ se realizó un primer Análisis Factorial Exploratorio y un segundo Análisis Factorial Confirmatorio. Se emplearon tres Análisis de Varianza (ANOVA) de dos vías para evaluar las diferencias en motivos entre los niveles de gravedad y tipo de actividad. De acuerdo con la estructura factorial del GMQ, existen tres principales grupos de motivaciones para jugar: búsqueda de emociones positivas, afrontamiento del estrés y causas sociales. En comparación con los jugadores sin problema, los jugadores problemáticos puntuaron más alto en todos los motivos. Además, los jugadores que buscan emociones positivas se decantan más por los juegos estratégicos, los que utilizan el juego para afrontar el estrés utilizan más los no estratégicos, mientras que los jugadores sociales no presentan preferencia por ninguno de los dos. En conclusión, la motivación para jugar se encuentra en relación con la gravedad y las características del juego. Estos resultados son de utilidad desde el punto de vista de la prevención.

Palabras clave: Juego de apuestas; Adolescentes; Motivos de juego; Juego problema.

\begin{abstract}
Self-reported reasons for gambling have been highlighted as crucial to understanding why adults develop problems with gambling. However, research on motives among adolescents remains scarce. The aim of this study is to explore the factorial structure of the Gambling Motives Questionnaire (GMQ) and to analyze differences in motives among adolescents, depending on the gambling activity and level of gambling severity. A total of 698 adolescent gamblers $(\mathrm{M}=15.24$, SD $=.76$ ) were assessed. As well as the GMQ, the South Oaks Gambling Screen (SOGS-RA) questionnaire was used. Gamblers were classified into three categories (strategic, non-strategic, and mixed) according to the activities engaged in during the last year. An Exploratory Factor Analysis was conducted followed by a Confirmatory Factor Analysis in order to explore the internal structure of the GMQ. Three twoway between-groups Analyses of Variance (ANOVA) were conducted to explore differences in motives according to the type of activity and the gambling severity levels. According to the factorial structure of the GMQ three main groups of motivations were found: enhancement, coping and social. Compared to non-problem gamblers, problem gamblers scored higher on all motives. Moreover, gamblers seeking enhancement prefer strategic games, those gambling to cope with stress use non-strategic games more frequently, while social gamblers do not show a preference for either strategic or non-strategic games. In conclusion, gambling motives are related to gambling severity and structural characteristics of gambling. These findings may be useful from a prevention standpoint.

Keywords: Gambling; Adolescents; Gambling motives; Problem gambling.
\end{abstract}

Recibido: Octubre 2017; Aceptado: Febrero 2018

Enviar correspondencia a:

Aris Grande Gosende, Addictive Behavior Research Group. Faculty of Psychology. University of Oviedo. Plaza Feijóo s/n, 33003, Oviedo (Asturias, Spain). Tel.: 0034985104 189. Email: grandearis@uniovi.es 
A pesar de ser una actividad ilegal, los juegos de apuestas se han vuelto muy populares entre los adolescentes, con tasas más altas de problemas relacionados con los mismos que en los adultos (Blinn-Pike, Worthy y Jonkman, 2010; Calado, Alexandre y Griffiths, 2016; Delfabbro, King y Derevensky, 2016). Los motivos autoinformados para jugar han sido identificados como variables clave para entender por qué los adultos desarrollan problemas con el juego (Moragas et al., 2015; Myrseth y Notelaers, 2017; Stewart, Zack, Collins y Klein, 2008). Sin embargo, la investigación sobre los motivos entre adolescentes sigue siendo muy escasa, con sólo un estudio realizado en la población de esta edad (Cerdà Salom, Nebot Ibáñez, Campos Bacas y Quero Castellano, 2016).

Los instrumentos diseñados para evaluar los motivos de juego en población adulta han sido diversos. Adaptado del "Drinking Motives Questionnaire" (DMQ-Cuestionario de Motivos para Beber; Cooper, Russell, Skinner y Windle, 1992), el "Gambling Motives Questionnaire" (GMQ- Cuestionario de Motivos de Juego (Stewart y Zack, 2008) es uno de los instrumentos más ampliamente utilizados. El GMQ muestra buenas propiedades psicométricas, como alta consistencia interna y validez concurrente para la predicción de conductas de juego excesivas y problemas de juego, y una estructura factorial similar a la de las motivaciones subyacentes al uso de otras sustancias adictivas, como el alcohol (Dechant y Ellery, 2011, Stewart y Zack, 2008). El GMQ original ofrece un modelo tridimensional de la motivación: (1) búsqueda de emociones positivas (en inglés "enhancement", ENH), que se refiere al refuerzo positivo interno; (2) afrontamiento de estrés (en inglés, "coping", COP), en términos de refuerzo interno negativo, que indica el uso del juego como estrategia de afrontamiento desadaptativa para escapar de estados emocionales negativos; y (3) motivos sociales (en inglés, "social”, SOC), relacionados con el refuerzo positivo externo (Stewart y Zack, 2008).

Sin embargo, este modelo ha sido recientemente cuestionado en adultos. Dado que ganar dinero ha sido señalada como una razón principal para jugar (Chantal, Vallerand y Vallieres, 1995; Myrseth y Notelaers, 2017; Stewart et al., 2008), algunos autores han postulado un motivo financiero adicional, añadiendo nuevos ítems (Dechant, 2014; Dechant y Ellery, 2011). Además, Myrseth y Notelaers (2017) revisaron el GMQ original, encontrando un cuarto motivo llamado autogratificación, definido como jugar para sentirse más seguro de sí mismo. Ante esta controversia y teniendo en cuenta la ausencia de un análisis factorial en adolescentes, la necesidad de investigación en menores de edad es evidente. Hasta donde llega nuestro conocimiento, solamente el estudio anteriormente citado (Cerdà Salom et al., 2016) ha examinado la estructura factorial entre los jóvenes, encontrando el tradicional modelo motivacional de tres factores. Sin embargo, debido al pequeño tamaño muestral y al uso tanto de participantes menores como mayores de edad, los resultados deben interpretarse con precaución.

Se han relacionado diferentes motivaciones con problemas de juego en población adulta. Mientras que los motivos SOC son más frecuentemente referidos por jugadores no problemáticos (J-NO-PROB) (Dechant y Ellery, 2011; Lambe, Mackinnon y Stewart, 2015; Stewart y Zack, 2008), los motivos ENH y COP son más prevalentes entre jugadores problemáticos (J-PROB) (Dechant y Ellery, 2011; Lambe et al., 2015; McGrath, Stewart, Klein y Barrett, 2010; Myrseth y Notelaers, 2017; Stewart y Zack, 2008). Sólo dos estudios han explorado esta asociación en jóvenes. El primero (Gupta y Derevensky, 1998) encontró que es más probable que los J-PROB informen de más razones para apostar que los jugadores regulares y ocasionales. El segundo estudio (Gupta, Derevensky y Marget, 2004) relacionó las estrategias desadaptativas de COP con el juego excesivo. Por otra parte, se ha observado que adolescentes que presentan diagnóstico de "Internet Gaming Disorder" (IGD - Trastorno de Juego por Internet) y un perfil psicopatológico internalizante, parecen utilizar los videojuegos preferentemente como una estrategia de afrontamiento de emociones desagradables (Martín-Fernández et al., 2017). Sin embargo, de los dos estudios mencionados, ni Gupta y Derevensky (1998) ni Gupta et al. (2004) consideran el tipo de actividades que los jugadores, manifestando la necesidad de más estudios para esclarecer esta relación.

Se ha observado que los motivos de juego varían entre los adultos involucrados en diferentes actividades de juego. Los individuos que juegan por ENH tienden a practicar juegos de habilidad con más frecuencia (Chantal y Vallerand, 1996; Fang y Mowen, 2009; Potenza et al., 2001; Wardle et al., 2011), mientras que los que juegan por COP prefieren juegos no estratégicos (Fang y Mowen, 2009; McGrath et al., 2010; Moragas et al., 2015; Wardle et al., 2011). Existen datos contradictorios con respecto a los tipos de actividad de juego y la gravedad del mismo. Estudios previos han relacionado el juego no estratégico (Bonnaire, Bungener y Varescon, 2009; Grant, Odlaug, Chamberlain y Schreiber, 2012; Griffiths, Scarfe y Bellringer, 1999; Navas et al., 2017), el estratégico (Moragas et al., 2015) y ambos tipos de actividad (Odlaug, Marsh, Won Kim y Grant, 2011) con mayor gravedad de juego. A pesar de la relevancia de estos hallazgos para la prevención y el tratamiento, ninguna investigación hasta la fecha ha abordado este tema entre los adolescentes.

El objetivo de este estudio fue doble. El primer objetivo fue explorar la estructura factorial y estimar la fiabilidad de las puntuaciones del GMQ en una muestra de adolescentes. Se hipotetizó que el análisis factorial replicaría la estructura clásica de la escala original, revelando su modelo tridimensional (Dechant y Ellery, 2011). El segundo objetivo fue analizar las diferencias en motivos de juego entre los participantes involucrados en diferentes actividades de juego y con diferentes niveles de gravedad del mismo. Se 
hipotetizó que los juegos estratégicos se relacionarían más con los motivos ENH, y los juegos no estratégicos con los motivos COP. Con respecto a los niveles de gravedad del juego, se esperaba que los J-PROB puntuaran más alto tanto en los motivos COP como los ENH, según lo indicado en investigaciones previas.

\section{Método}

Se han seguido los estándares de rigor metodológico en el estudio de las adicciones en la redacción de este artículo (Fonseca Pedrero, 2017).

\section{Participantes}

Se evaluó un total de 1.810 adolescentes de edades comprendidas entre 14 y 17 años de 22 escuelas españolas de enseñanza secundaria (16 del Principado de Asturias y 6 de Alicante), tanto públicas como concertadas. Los centros se seleccionaron siguiendo un procedimiento aleatorio estratificado e incidental. Los criterios de exclusión preestablecidos fueron: (1) tener alguna discapacidad sensorial o intelectual $(n=1)$, (2) presentar dificultades con el idioma

Tabla 1. Características de los participantes

\begin{tabular}{|c|c|c|}
\hline Datos sociodemográficos & $n$ & $\%$ \\
\hline Sexo (varón) & 436 & 62,5 \\
\hline Edad $_{1}$ & 698 & $15,23(, 74)$ \\
\hline Nacionalidad (español) ${ }_{2}$ & 586 & 90,7 \\
\hline $\begin{array}{l}\text { Estructura familiar } \\
\text { No vive con los padres } \\
\text { Familia monoparental } \\
\text { Vive con ambos padres }\end{array}$ & $\begin{array}{r}15 \\
177 \\
506\end{array}$ & $\begin{array}{r}2,1 \\
25,4 \\
72,5\end{array}$ \\
\hline $\begin{array}{l}\text { Asignación semanal } \\
\begin{array}{l}0-10 € \\
10-20 € \\
20-40 € \\
\text { Más de } 40 €\end{array}\end{array}$ & $\begin{array}{r}301 \\
290 \\
81 \\
26\end{array}$ & $\begin{array}{r}43,1 \\
41,5 \\
11,6 \\
3,7\end{array}$ \\
\hline $\begin{array}{l}\text { Nota académica más frecuente } \\
\text { Suspenso } \\
\text { Aprobado } \\
\text { Notable } \\
\text { Sobresaliente }\end{array}$ & $\begin{array}{r}100 \\
250 \\
225 \\
71\end{array}$ & $\begin{array}{r}15,5 \\
38,7 \\
34,8 \\
11\end{array}$ \\
\hline $\begin{array}{l}\text { Tipo de actividad de juego } \\
\text { Jugadores estratégicos } \\
\text { Jugadores no estratégicos } \\
\text { Jugadores mixtos }\end{array}$ & $\begin{array}{l}162 \\
329 \\
207\end{array}$ & $\begin{array}{l}23,2 \\
47,1 \\
29,7\end{array}$ \\
\hline $\begin{array}{l}\text { Gravedad de juego } \\
\text { Jugadores problemáticos }\end{array}$ & 101 & 14,5 \\
\hline $\begin{array}{l}\text { Motivos de juego }_{3} \\
\text { Enhancement (Emociones positivas) } \\
\text { Coping (Afrontamiento) } \\
\text { Social (Motivos sociales) }\end{array}$ & $\begin{array}{l}79 \\
25 \\
49\end{array}$ & $\begin{array}{r}11,3 \\
3,6 \\
7\end{array}$ \\
\hline
\end{tabular}

Nota. 1 = media (desviación típica), 2 = 52 datos ausentes debido a problemas técnicos en la recolección de datos, 3 = se muestra la frecuencia de jugadores que informaron jugar a menudo o siempre en cada subescala del Cuestionario de Motivos de Juego. español $(n=0)$, (3) tener 18 años o más $(n=11)$ y (4) presentar respuestas al azar $(n=43)$. Debido a estos criterios, 55 participantes fueron eliminados del estudio y 1 más debido a problemas técnicos. Tras haber eliminado a los participantes que no habían jugado en el último año $(n$ $=1,056)$, la muestra final se compuso de 698 adolescentes. Las características de los participantes se muestran en la Tabla 1.

\section{Instrumentos}

Variables de control. Se utilizó la Escala de Infrecuencia de Oviedo - INF-OV (Fonseca-Pedrero, Paíno, Lemos-Giráldez y Muñiz, 2008) para excluir a los participantes que presentaron respuestas al azar. Sus 12 ítems de tipo Likert sobre hechos evidentes (por ejemplo, "Conozco personas que usan gafas", "A veces he visto películas en la televisión”) se entremezclaron a lo largo de todo el cuestionario. De acuerdo con las recomendaciones de los autores, se excluyeron a los participantes que presentaron más de tres respuestas incorrectas.

Motivos de juego. Se utilizó una versión en española del GMQ (Dechant y Ellery, 2011) (véase el Apéndice). Los participantes debían indicar en una escala tipo Likert (1 = nunca o casi nunca, 4 = casi siempre o siempre) con qué frecuencia jugaron por cada motivo. El GMQ proporciona una puntuación para cada subescala, obteniéndose un perfil motivacional del jugador. Las subescalas no son excluyentes, por lo tanto, cada jugador puede puntuar en todas ellas. Después de realizar un procedimiento de "traducción y retrotraducción" de la versión original al español, se llevaron a cabo las correspondientes adaptaciones lingüísticas y culturales siguiendo la segunda edición de las directrices de la International Test Commission (ITC) para adaptar pruebas a otras culturas (Muñiz, Elosua y Hambleton, 2013). Se verificaron las directrices de calidad para la traducción y adaptación de los ítems propuestas por Hambleton y Zenisky (2011) para el cuestionario completo. La versión original demostró buena fiabilidad de las puntuaciones para cada factor (ENH: $\alpha=0,74$; COP: $\alpha=0,76$; SOC $\alpha=0,67$ ).

Actividades de juego. Los participantes indicaban la frecuencia con que habían jugado en el último año antes de la evaluación en diferentes actividades, tanto de forma presencial como online (bingo, póquer, otros juegos de casino [OJCs], apuestas deportivas, lotería, rascas y máquinas tragaperras $[\mathrm{MTs}])$. Antes de realizar los análisis, las actividades fueron clasificadas según sus características siguiendo a Moragas et al. (2015). En base a esto, los jugadores que participaron en actividades que ponen de manifiesto las habilidades individuales (OJCs, póquer y apuestas deportivas) se clasificaron como jugadores estratégicos (JST), mientras que los participantes que apostaron en juegos que implican el azar (MTs, bingo, lotería o rascas) se consideraron como jugadores no estratégicos (J-NST). Los que 
participaban en ambos tipos de juego fueron clasificados como jugadores mixtos (JMX).

Gravedad del Juego. La gravedad del juego se evaluó por medio de la adaptación española (Becoña, 1997) del South Oaks Gambling Screen-Revised for Adolescents (SOGS-RA - Instrumentos para el Cribado de Juego - Revisado para Adolescentes - Winters, Stinchfield y Fulkerson, 1993). El SOGS-RA tiene 10 ítems dicotómicos $($ no = 0 , sí = 1) y evalúa los problemas relacionados con los juegos de azar en el pasado año. Los individuos pueden clasificarse en tres categorías: no problemático (0-1 puntos), en riesgo (2-3) y jugadores problemáticos (4 o más). La versión en español demostró buena fiabilidad de las puntuaciones $(\alpha=0,80)$.

\section{Procedimiento}

Se encuestaron a los alumnos en sus propias aulas con dispositivos digitales (Samsung Galaxy Tab2 10.1). El software fue diseñado para impedir respuestas inadecuadas basadas en respuestas anteriores y para detectar respuestas en blanco, y recordando a los participantes que revisasen sus respuestas para evitar datos perdidos. Los estudiantes completaron la encuesta en una sola sesión de 75 minutos. Un investigador especialmente entrenado para ello daba las instrucciones para completar los cuestionarios y supervisaba el procedimiento. La participación en el estudio fue completamente voluntaria y no se ofreció ninguna compensación. Antes de la evaluación, se obtuvo el consentimiento informado de las escuelas, los padres y las autoridades educativas. Se informó a los estudiantes sobre la confidencialidad y el anonimato de los datos. Todos los participantes dieron su consentimiento informado y nadie se negó a participar en el estudio. Se obtuvo la aprobación ética de la Comisión de Investigación de la Universidad de Oviedo.

\section{Análisis de datos}

Se realizaron análisis descriptivos para evaluar las características sociodemográficas y de juego. Se exploraron las puntuaciones atípicas ("outliers") en el GMQ y se modificaron siguiendo a Tabachnick y Fidell (2007). Para el estudio de las fuentes de validez basada en la evidencia de la estructura interna, se realizó un análisis factorial exploratorio (AFE) inicial Promin y después un análisis factorial confirmatorio (AFC) Procrustes, empleando la estimación robusta de mínimos cuadrados no ponderados (RULS), dividiendo la muestra al azar en dos submuestras. El número de factores se determinó por el criterio bayesiano de información de Schwarz (BIC). Se utilizaron el índice de ajuste comparativo (CFI) y la media cuadrática de los residuales (RMSR) para determinar la bondad de ajuste, considerando CFI > ,98 y RMSR < ,05 como valores de referencia. Se realizó el análisis de fiabilidad de las puntuaciones mediante el alfa de Cronbach y el omega de McDonald. Aunque el alfa de Cronbach es el índice más utilizado para estimar la consistencia interna, omega de McDonald (1999) se re- comienda para ítems de respuesta ordinal o escalas tipo Likert con menos de cinco categorías (Elosua Oliden y Zumbo, 2008). Además, la omega se ha considerado como un índice de consistencia interna más sensible y ha mostrado menor riesgo de sobreestimación o subestimación de la fiabilidad de las puntuaciones (Dunn, Baguley y Brunsden, 2014). Después de un estudio preliminar de la equivalencia de las propiedades psicométricas del GMQ, atendiendo al sexo, edad y niveles de gravedad del juego, se llevaron a cabo pruebas- $t$ para evaluar las diferencias en los motivos de juego según sexo y edad. Con el fin de explorar las diferencias en los motivos de juego según la gravedad y el tipo de juego, se llevaron a cabo tres análisis de varianza de dos vías entre grupos (ANOVA). Se emplearon las comparaciones de pares GT2 de Hochberg para ajustar los grupos no balanceados. Debido al pequeño tamaño de la muestra de cada categoría SOGS-RA y a los problemas asociados con el juego de riesgo y el juego problemático (Potenza et al., 2001), los participantes fueron clasificados en dos grupos: J-NO-PROB (SOGS-RA $\leq 1$ ) y J-PROB (SOGS-RA $\geq 2$ ). Se realizaron los análisis factoriales con el software FACTOR 10.4.01 (Ferrando y Lorenzo-Seva, 2017) y los análisis descriptivos y análisis de varianza con el SPSS 22.0.

\section{Resultados}

\section{Estructura factorial del GMQ}

En primer lugar, se llevó a cabo un AFE en la primera submuestra $(n=347)$. La prueba Kaiser-Meyer-Olkin y la prueba de esfericidad de Bartlett mostraron una buena adecuación para el análisis factorial $\left(\mathrm{KMO}=, 875 ; \chi_{(120)}^{2}=2411,9\right.$, $p<, 001)$. El BIC recomendó la extracción de tres factores, explicando el $66,8 \%$ de la varianza $(43,1 \%$ Factor $1 ; 12,3 \%$ Factor 2; y 11,4\% Factor 3). El índice de ajuste comparativo $(\mathrm{CFI}=, 993)$ y la media cuadrática de residuos $(\mathrm{RMSR}=, 036)$ mostraron un excelente ajuste al modelo. Se realizó un AFC en la segunda submuestra $(n=351)$ con la estructura recomendada $\left(\mathrm{KMO}=, 899 ; \chi^{2}{ }_{(120)}=3177,7, p<, 001 ; \mathrm{CFI}=, 986\right.$; RMSR = ,038), que explicó el $67,7 \%$ de la varianza $(48,6 \%$ Factor 1; 10,6\% Factor 2; y 8,5\% Factor 3). Las cargas de los ítems confirmaron la estructura clásica tridimensional. Las cargas factoriales oscilaron entre 0,43 y 0,93 para el Factor 1 (SOC), de 0,71 a 0,95 para el Factor $2(\mathrm{ENH})$ y de 0,45 a 0,91 para el Factor 3 (COP) (ver Tabla 2). El ítem financiero (ítem 16) cargó menos de 0,3 en todos los factores, indicando su inadecuación para el modelo.

\section{Estimación de la fiabilidad de las puntuaciones}

Los alfa de Cronbach mostraron una buena consistencia interna para las tres subescalas (ENH: $\alpha=, 85$; COP: $\alpha=, 87$; SOC: $\alpha=, 80)$, además de la omega de McDonald (ENH: $\omega=, 86$; COP: $\omega=, 87$; SOC: $\omega=, 81)$. La correlación entre factores fue adecuada $\left(r_{\mathrm{ENH}-\mathrm{COP}}=0,659 ; r_{\mathrm{ENH}-\mathrm{SOC}}=0,623 ; r_{\mathrm{COP}-}\right.$ $\operatorname{sOC}=0,627)$. 
Tabla 2. Estadísticos Descriptivos y Cargas Factoriales de cada Ítem en las Subescalas del Cuestionario de Motivos de Juego

\begin{tabular}{|c|c|c|c|c|c|}
\hline İtems & $M(D T)$ & $\begin{array}{c}\text { Índices de } \\
\text { discriminación } \\
\text { de los ítems }\end{array}$ & ENH & COP & SOC \\
\hline 3. Porque te gusta lo que sientes & $0,68(0,86)$ & 0,71 & 0,92 & & \\
\hline 6. Porque es excitante & $0,48(0,78)$ & 0,73 & 0,95 & & \\
\hline 15. Porque te hace sentir bien & $0,5(0,80)$ & 0,75 & 0,81 & & \\
\hline 12. Porque es divertido & $1,31(1,06)$ & 0,55 & 0,68 & & \\
\hline 9. Para conseguir un sentimiento intenso o "de subidón" & $0,39(0,73)$ & 0,62 & 0,71 & & \\
\hline 5. Para olvidar las preocupaciones & $0,23(0,58)$ & 0,7 & & 0,69 & \\
\hline 11. Porque te ayuda cuando te sientes nervioso o deprimido & $0,21(0,54)$ & 0,77 & & 0,90 & \\
\hline 14. Para levantarte el ánimo cuando te sientes mal & $0,24(0,59)$ & 0,74 & & 0,91 & \\
\hline 8. Porque te sientes más seguro de ti mismo & $0,25(0,63)$ & 0,64 & & 0,54 & \\
\hline 2. Para relajarte & $0,4(0,7)$ & 0,64 & & 0,46 & \\
\hline 7. Para ser sociable & $0,27(0,65)$ & 0,51 & & & 0,43 \\
\hline 4. Porque es lo que la mayoría de tus amigos hace cuando se juntan & $0,38(0,71)$ & 0,54 & & & 0,77 \\
\hline 13. Porque esto hace que una reunión de amigos sea más agradable & $0,52(0,79)$ & 0,69 & & & 0,93 \\
\hline 1. Como una manera de celebración & $0,46(0,79)$ & 0,66 & & & 0,65 \\
\hline 10. Porque es algo que haces en ocasiones especiales & $0,71(0,9)$ & 0,56 & & & 0,5 \\
\hline
\end{tabular}

Nota. Se han omitido las cargas factoriales < 0,30. M (DT) = Media (Desviación Típica); ENH: Enhancement motive (Emociones positivas); COP: Coping motive (Afrontamiento); SOC: Social motive (Motivos sociales).

Tabla 3. Diferencias en Motivos de Juego según Sexo y Edad

\begin{tabular}{|c|c|c|c|c|c|c|}
\hline \multirow[t]{3}{*}{ Motivos de Juego } & \multicolumn{2}{|c|}{ Sexo } & \multicolumn{2}{|c|}{ Edad } & \multirow[t]{3}{*}{$t$} & \multirow[t]{3}{*}{$\eta^{2}$} \\
\hline & \multicolumn{2}{|c|}{$M(D T)$} & \multicolumn{2}{|c|}{$M(D T)$} & & \\
\hline & $\begin{array}{l}\text { Chicos } \\
(n=436)\end{array}$ & $\begin{array}{c}\text { Chicas } \\
(n=262)\end{array}$ & $\begin{array}{c}14-15 \text { años } \\
(n=503)\end{array}$ & $\begin{array}{c}\text { 16-17 años } \\
(\mathrm{n}=195)\end{array}$ & & \\
\hline ENH & $3,64(3,55)$ & $2,88(2,99)$ & & & $3,05^{\star}$ & 0,01 \\
\hline COP & $1,46(2,4)$ & $0,96(2,01)$ & & & $2,92^{*}$ & 0,01 \\
\hline SOC & $2,5(2,94)$ & $2,06(2,57)$ & & & $2,1^{\star}$ & 0,01 \\
\hline ENH & & & $3,35(3,39)$ & $3,38(3,34)$ & $-0,11$ & - \\
\hline COP & & & $1,28(2,32)$ & $1,28(2,13)$ & $-0,3$ & - \\
\hline SOC & & & $2,47(2,89)$ & $1,98(2,58)$ & $2,09^{\star}$ & 0,01 \\
\hline
\end{tabular}

Nota. ENH: Enhancement motive (Emociones positivas); COP: Coping motive (Afrontamiento); SOC: Social motive (Motivos sociales). M (DT) = Media (Desviación Típica). ${ }^{*} p<0,05$.

Tabla 4. Motivos de juego según niveles de gravedad

\begin{tabular}{lcccc}
\hline $\begin{array}{l}\text { Motivos } \\
\text { de juego }\end{array}$ & $\begin{array}{c}\text { J-PROB } \\
(\boldsymbol{n}=\mathbf{1 0 1})\end{array}$ & $\begin{array}{c}\text { J-NO-PROB } \\
(\boldsymbol{n}=\mathbf{5 9 7})\end{array}$ & $\boldsymbol{F}$ & $\eta$ parcial $^{2}$ \\
\hline & $\mathrm{M}(\mathrm{SD})$ & $\mathrm{M}(\mathrm{SD})$ & & \\
ENH & $5,42(4,37)$ & $3,00(3,04)$ & $34,95^{\star *}$ & 0,05 \\
COP & $2,87(3,27)$ & $1,01(1,93)$ & $50,7^{\star *}$ & 0,07 \\
SOC & $3,72(3,66)$ & $2,10(2,58)$ & $21,56 * *$ & 0,03 \\
\hline
\end{tabular}

Nota. J-PROB: Jugadores problemáticos; NO-PROB: Jugadores no problemáticos; ENH: Enhancement motive (emociones positivas); COP: Coping motive (Afrontamiento); SOC: Social motive (Motivos sociales).

${ }^{\star \star} p<0,001$.

\section{Diferencias en los motivos de juego}

Se exploraron las diferencias en los motivos según sexo y edad, mostrando los varones puntuaciones más altas que las mujeres en las tres subescalas. Los jugadores de 14 y 15 años de edad reflejaron mayores niveles de motivos SOC que los jugadores con edades entre 16-17 años (ver Tabla 3 ).

Se analizaron las diferencias según la gravedad y el tipo de actividad de juego. El efecto principal de la gravedad del juego fue significativo en $\mathrm{ENH}\left(F_{(1,692)}=34,95, p<, 001\right.$, $\eta^{2}$ parcial $\left.=, 05\right)$, con los J-PROB puntuando más alto que los J-NO-PROB (ver Tabla 4). El tipo de juego también fue significativo $\left(F_{(2,692)}=4,23, p=, 015, \eta^{2}\right.$ parcial $\left.=, 01\right)$. En concreto, los J-NST puntuaron más bajo en ENH que los 
Tabla 5. Motivos de Juego según el Tipo de Juego

\begin{tabular}{|c|c|c|c|c|c|}
\hline & $\begin{array}{c}\text { JST } \\
(n=162)\end{array}$ & $\begin{array}{c}\text { J-NST } \\
(n=329)\end{array}$ & $\begin{array}{c}\text { JMX } \\
(n=207)\end{array}$ & $F$ & $\eta$ parcial $^{2}$ \\
\hline & $M \pm D T$ & $M \pm D T$ & $M \pm D T$ & & \\
\hline ENH & $3,77 \pm 3,57 a$ & $2,68 \pm 3,04 b$ & $4,09 \pm 3,52 a$ & $4,23^{*}$ & 0,01 \\
\hline \multicolumn{6}{|l|}{ COP } \\
\hline J-PROB & $1,68 \pm 2,23 a$ & $3,6 \pm 4,04$ & $3,04 \pm 3,06$ & $6,16^{*}$ & 0,02 \\
\hline J-NO-PROB & $1,11 \pm 2,1_{a}$ & $0,84 \pm 1,86_{a}$ & $1,24 \pm 1,9_{\mathrm{a}}$ & $6,16^{*}$ & 0,02 \\
\hline SOC & $2,12 \pm 2,53 \mathrm{a}$ & $1,98 \pm 2,73 \mathrm{a}$ & $3,06 \pm 3,02_{b}$ & $4,36^{*}$ & 0,01 \\
\hline
\end{tabular}

Nota. Los subíndices indican diferencias entre los grupos. Grupos con el mismo subíndice no difirieron significativamente entre sí. J-PROB: Jugadores problemáticos; J-NO-PROB: Jugadores no problemáticos; JST: Jugadores estratégicos; J-NST: Jugadores no estratégicos; JMX: Jugadores mixtos; ENH: Enhancement motive (Emociones positivas); COP: Coping motive (Afrontamiento); SOC: Social motive (Causas sociales); M (DT) = Media (Desviación Típica).

${ }^{*} p<0,05$.

JST $(p=, 001)$ y los JMX $(p<, 001)$ (ver Tabla 5$)$. El efecto de interacción no fue significativo $\left(F_{(1,692)}=, 40, p<, 67, \eta^{2}\right.$ parcial $=, 00)$.

El efecto de interacción entre la gravedad y tipo de juego fue significativo en $\operatorname{COP}\left(F_{(2,692)}=6,16, p=, 002, \eta^{2}\right.$ parcial $=, 02)$. Específicamente, los J-PROB no estratégicos y mixtos obtuvieron puntuaciones más altas en COP que los J-PROB estratégicos $(p=, 003$ y $p=, 033$, respectivamente) (ver la Tabla 5).

El efecto principal de la gravedad del juego fue significativo en SOC $\left(F_{(1,692)}=21,56, p<, 001, \eta^{2}\right.$ parcial $\left.=, 03\right)$, con los J-PROB puntuando más alto que los J-NO-PROB (ver Tabla 4). El tipo de juego también fue significativo $\left(F_{(2,692)}\right.$ $=4,36, p=, 013, \eta^{2}$ parcial $\left.=, 01\right)$, con los JMX mostrando mayor SOC que los JST $(p=, 003)$ y los J-NST $(p, 001)$ (ver Tabla 5). El efecto de interacción no fue significativo $\left(F_{(2,}\right.$ ${ }_{692)}=, 77, p=, 462, \eta^{2}$ parcial $\left.=, 00\right)$.

\section{Discusión}

Este es el primer estudio destinado a explorar las diferencias en los motivos de juego entre diferentes actividades de apuestas y la gravedad del juego en adolescentes. Se han alcanzado tres conclusiones principales: 1) se encontraron tres motivos principales para jugar, clasificados en los siguientes grupos de factores: la búsqueda de emociones positivas (ENH), el afrontamiento de estrés (COP) y motivos sociales (SOC); 2) Los jugadores problemáticos (J-PROB) obtuvieron puntuaciones más altas en todos los motivos para jugar; 3) Mientras que los jugadores estratégicos (JST) puntuaron más alto ENH y los J-PROB no estratégicos (J-NST) puntuaron más alto en COP, los jugadores SOC tendieron a involucrarse tanto en juegos estratégicos como no estratégicos.

De acuerdo con la estructura tradicional (Stewart y Zack, 2008), los resultados sobre la evidencia de estructura interna del GMQ mostraron una solución de tres factores. En este estudio realizado en menores de edad, no se encontró ninguna evidencia del motivo financiero recientemente señalado en adultos (Dechant, 2014; Dechant y Ellery,
2011). En contraste con la población adulta, ganar dinero no parece ser una razón substancial para jugar en los adolescentes. Tal y como señaló Zuckerman (1994), el riesgo y la incertidumbre asociados a las apuestas y la potencial pérdida o ganancia pueden ser altamente excitantes. Así, los adolescentes pueden apostar más por el impulso de ganar y la emoción del juego que para ganar dinero en sí mismo (Derevensky y Gilbeau, 2015).

En cuanto a gravedad de juego, los adolescentes J-PROB reflejaban puntuaciones más altas en los tres motivos de juego. Consistente con investigaciones anteriores en población adulta (Dechant y Ellery, 2011; Lambe et al., 2015; McGrath et al., 2010; Myrseth y Notelaers, 2017; Stewart y Zack, 2008) y adolescente (Gupta y Derevensky, 1998; Gupta et al., 2004), los J-PROB puntuaron más alto ENH y en COP. Los adolescentes J-PROB mostraron niveles más altos en SOC, al igual que en el único estudio que aborda este tema en esta población (Gupta y Derevensky, 1998). Este hallazgo contrasta con la investigación del juego en adultos, que relaciona el motivo SOC con los J-NO-PROB (Dechant y Ellery, 2011; Lambe et al., 2015; Stewart y Zack, 2008). Dicha inconsistencia puede tener varias explicaciones. La expansión de espacios de juego, la mayor variedad de actividades de apuestas y el efecto de la publicidad en los medios de comunicación podría llevar a los adolescentes a considerar el juego como una actividad de ocio aceptable (St-Pierre, Walker, Derevensky y Gupta, 2014). Esto podría contribuir a aumentar la popularidad de los juegos de azar como un medio para la socialización entre los iguales (Derevensky 2012; Derevensky y Gilbeau, 2015), ya que los adolescentes son más sensibles que los adultos a estos estímulos. Así, las intervenciones preventivas deberían orientarse hacia estrategias de ocio responsable y saludable para evitar la identificación de los juegos de azar como una actividad de entretenimiento de bajo riesgo.

Con respecto a los tipos de actividad de juego, se encontraron tres resultados principales. En primer lugar, los JST puntuaron más alto en ENH que los J-NST, en consonancia con la evidencia previa en adultos (Chantal y Vallerand, 
1996; Fang y Mowen, 2009; McGrath et al., 2010). El motivo ENH se basa en el aumento de las emociones positivas y se relaciona con la búsqueda de sensaciones. Aquellas personas que muestran este rasgo tienden a buscar "experiencias nuevas, variadas o sensaciones complejas [... y] están dispuestos a arriesgarse para alcanzar esta experiencia" (Breen y Zuckerman, 1999). Investigaciones previas han relacionado los juegos estratégicos o activos con este constructo de personalidad (Bonnaire et al., 2009; Bonnaire, Bungener y Varescon, 2017), ya que se perciben como actividades que son fácilmente dominadas a través del conocimiento (Breen y Zimmerman, 2002). Estos juegos tienden a demorar el resultado de las apuestas durante horas, lo que implica una mayor planificación y la regulación de estados fisiológicos de hipo-excitación (Bonnaire et al., 2009; Cocco, Sharpe y Blaszczynski, 1995) aspectos que encajan mejor con el factor motivacional ENH que con los demás. En segundo lugar, los J-PROB no estratégicos (J-NST) puntuaron más alto en COP que los J-PROB estratégicos (JST). Este hallazgo se apoya en las investigaciones anteriores, tanto en adultos (Bonnaire et al., 2009; Grant et al., 2012; Navas et al., 2017) como en adolescentes (Bergevin, Gupta, Derevensky y Kaufman, 2006; Gupta et al., 2004). La presentación de un patrón de juego excesivo y problemático ha sido identificada como una estrategia de afrontamiento evitativa e inadecuada (Bergevin et al., 2006; Gupta et al., 2004). Por lo tanto, los J-PROB adolescentes en búsqueda del alivio de estados emocionales internos negativos suelen preferir juegos de azar con patrones continuos y repetitivos (Breen y Zimmerman, 2002; Dickerson, 1993). Finalmente, los jugadores adolescentes SOC tienden a utilizar tanto los juegos estratégicos como los no estratégicos, sin ningún tipo de preferencia. Este tipo de jugador está motivado por el refuerzo externo (es decir, la interacción social con sus semejantes). En este sentido, los jugadores SOC tienden a dejarse llevar por las circunstancias sociales y las oportunidades de jugar, recurriendo tanto a juegos estratégicos como no estratégicos indiscriminadamente. Cabe destacar que aquellos que participaron en ambos tipos de juegos (JMX) obtuvieron las puntuaciones más altas de los tres grupos. Este resultado puede indicar que los JMX son un grupo heterogéneo compuesto por diversas subpoblaciones de jugadores. De este modo, estudios futuros deberían poner atención en este grupo de jugadores para determinar mejor sus características.

No obstante, y a pesar de lo expuesto anteriormente, este trabajo no se encuentra exento de ciertas limitaciones. En primer lugar, el diseño transversal del estudio impide el establecimiento de efectos causales. El desarrollo de diseños longitudinales que exploren los patrones de juego en la adolescencia podrían encontrar cambios en las propias motivaciones de juego. En segundo lugar, el uso de medidas autoinformadas puede dar lugar a datos sesgados. Sin embargo, la utilización de un sistema informatizado permitió prevenir respuestas inconsistentes basadas en respues- tas anteriores, así como garantizar una alta fiabilidad de las puntuaciones. A pesar de estas limitaciones, el presente estudio contribuye a ampliar la investigación de los aspectos motivacionales de juego, proporcionando resultados novedosos en el campo de la investigación del juego en adolescentes.

En conclusión, el estudio de los motivos de juego en adolescentes a través del GMQ podría ser útil para la evaluación e implementación de estrategias preventivas, así como para el diseño de los tratamientos. Este estudio muestra la relevancia de los niveles de gravedad de juego y el tipo de actividad en las motivaciones de juego.

\section{Agradecimientos}

Los autores desean agradecer a las autoridades educativas, las escuelas, el personal y los estudiantes por su participación en el estudio.

Este estudio fue financiado por el Ministerio de Economía y Trabajo (FC-15-GRUPIN14-047) y por una Beca Predoctoral BP16071 del Ministerio de Educación y Cultura del Principado de Asturias (España). Las fuentes de financiación no desempeñaron ningún papel en el diseño del estudio, la recogida, análisis o interpretación de los datos, en la redacción del manuscrito o la decisión de enviar el artículo para su publicación.

\section{Conflicto de intereses}

Los autores declaran no tener conflicto de intereses.

\section{Referencias}

Becoña, E. (1997). Pathological gambling in Spanish children and adolescents: an emerging problem. Psychological Reports, 81, 275-287. doi:10.2466/pr0.1997.81.1.275.

Bergevin, T., Gupta, R., Derevensky, J. y Kaufman, F. (2006). Adolescent gambling: understanding the role of stress and coping. Journal of Gambling Studies, 22, 195208. doi:10.1007/s10899-006-9010-z.

Blinn-Pike, L., Worthy, S. L. y Jonkman, J. N. (2010). Adolescent gambling: a review of an emerging field of research. Journal of Adolescent Health, 47, 223-236. doi:10.1016/j.jadohealth.2010.05.003.

Bonnaire, C., Bungener, C. y Varescon, I. (2009). Subtypes of French pathological gamblers: comparison of sensation seeking, alexithymia and depression scores. Journal of Gambling Studies, 25, 455-471. doi:10.1007/s10899009-9142-z.

Bonnaire, C., Bungener, C. y Varescon, I. (2017). Sensation seeking in a community sample of French gamblers: comparison between strategic and non-strategic gamblers. Psychiatry Research, 250, 1-9. doi:10.1016/j. psychres.2017.01.057. 
Breen, R. B. y Zimmerman, M. (2002). Rapid onset of pathological gambling in machine gamblers. Journal of Gambling Studies, 18, 31-43. doi:10.1023/A:1014580112648.

Breen, R. B. y Zuckerman, M. (1999). 'Chasing' in gambling behavior: personality and cognitive determinants. Personality and Individual Differences, 27, 1097-1111. doi:10.1016/S0191-8869(99)00052-5.

Calado, F., Alexandre, J. y Griffiths, M. D. (2016). Prevalence of adolescent problem gambling: a systematic review of recent research. Journal of Gambling Studies, 33, 397424. doi:10.1007/s10899-016-9627-5.

Cerdà Salom, C., Nebot Ibáñez, S., Campos Bacas, D. y Quero Castellano, S. (2016). Validación española del cuestionario de motivos para el juego (GMQ) en población general. Àgora de Salut, 3, 95-102. doi:10.6035/ AgoraSalut.2016.3.10.

Cocco, N., Sharpe, L. y Blaszczynski, A. P. (1995). Differences in preferred level of arousal in two sub-groups of problem gamblers: a preliminary report. Journal of Gambling Studies, 11, 221-229. doi:10.1007/BF02107116.

Cooper, M. L., Russell, M., Skinner, J. B. y Windle, M. (1992). Development and validation of a three-dimensional measure of drinking motives. Psychological Assessment, 4, 123-132. doi:10.1037/1040-3590.4.2.123.

Chantal, Y. y Vallerand, R. J. (1996). Skill versus luck: a motivational analysis of gambling involvement. Journal of Gambling Studies, 12, 407-418. doi:10.1007/BF01539185.

Chantal, Y., Vallerand, R. J. y Vallieres, E. F. (1995). Motivation and gambling involvement. The Journal of Social Psychology, 135, 755-763. doi:10.1080/00224545.1995.9713978.

Dechant, K. (2014). Show me the money: incorporating financial motives into the Gambling Motives Questionnaire. Journal of Gambling Studies, 30, 949-965. doi:10.1007/ s10899-013-9386-5.

Dechant, K. y Ellery, M. (2011). The effect of including a monetary motive item on the Gambling Motives Questionnaire in a sample of moderate gamblers. Journal of Gambling Studies, 27, 331-344. doi:10.1007/s10899-010-9197-x.

Delfabbro, P., King, D. L. y Derevensky, J. L. (2016). Adolescent gambling and problem gambling: prevalence, current issues, and concerns. Current Addiction Reports, 3, 268-274. doi:10.1007/s40429-016-0105-z.

Derevensky, J. L. (2012). Teen gambling: understanding a growing epidemic. New York, Rowman \& Littlefield Publishers.

Derevensky, J. L. y Gilbeau, L. (2015). Adolescent gambling: twenty-five years of research. Canadian Journal of Addiction, 6, 4-12.

Dickerson, M. G. (1993). Internal and external determinants of persistent gambling: problems in generalising from one form of gambling to another. Journal of Gambling Studies, 11, 249-263. doi:10.1007/BF01015920.

Dunn, T. J., Baguley, T. y Brunsden, V. (2014). From alpha to omega: a practical solution to the pervasive problem of internal consistency estimation. British Journal of Psychology, 105, 399-412. doi:10.1111/bjop.12046.

Elosua Oliden, P. y Zumbo, B. D. (2008). Coeficientes de fiabilidad para escalas de respuesta categórica ordenada. Psicothema, 20, 896-901.

Fang, X. y Mowen, J. C. (2009). Examining the trait and functional motive antecedents of four gambling activities: slot machines, skilled card games, sports betting, and promotional games. Journal of Consumer Marketing, 26, 121-131. doi:10.1108/07363760910940483.

Ferrando, P. J. y Lorenzo-Seva, U. (2017). Program FACTOR at 10: Origins, development and future directions. Psicothema, 29, 236-240. doi: 10.7334/psicothema2016.304

Fonseca-Pedrero, E. (2017). Rigor metodológico en el estudio de las adicciones. Adicciones, 29, 147-149. doi:10.20882/adicciones.994.

Fonseca-Pedrero, E., Paíno, M., Lemos-Giráldez, S. y Muñiz, J. (2008). Construction and validation of the Oviedo Infrequency Scale in Spanish adolescents. Doctoral dissertation, University of Oviedo, Spain.

Grant, J. E., Odlaug, B. L., Chamberlain, S. R. y Schreiber, L. R. (2012). Neurocognitive dysfunction in strategic and non-strategic gamblers. Progress in Neuro-Psychopharmacology and Biological Psychiatry, 38, 336-340. doi:10.1016/j. pnpbp.2012.05.006.

Griffiths, M., Scarfe, A. y Bellringer, P. (1999). The UK national telephone gambling helpline - Results on the first year of operation. Journal of Gambling studies, 15, 83-90. doi:10.1023/A:1023071113879.

Gupta, R. y Derevensky, J. L. (1998). An empirical examinination of Jacobs' general theory of addictions: Do adolescent gamblers fit the theory? Journal of Gambling Studies, 14, 17-49. doi:10.1023/A:1023046509031.

Gupta, R., Derevensky, J. y Marget, N. (2004). Coping strategies employed by adolescents with gambling problems. Child and Adolescent Mental Health, 9, 115-120. doi:10.1111/j.1475-3588.2004.00092.x.

Hambleton, R. K. y Zenisky, A. L. (2011). Translating and adapting tests for cross-cultural assessments. In D. Matsumoto y F. J. R. van de Vijver (Eds.), Cross-cultural research methods in psychology (46-70). New York: Cambridge University Press.

Lambe, L., Mackinnon, S. P. y Stewart, S. H. (2015). Validation of the Gambling Motives Questionnaire in emerging adults. Journal of Gambling Studies, 31, 867-885. doi:10.1007/s10899-014-9467-0.

Martín-Fernández, M., Matalí, J.L., García-Sánchez, S., Pardo, M., Lleras, M. y Castellano-Tejedor C. (2017). Adolescents with Internet Gaming Disorder (IGD): profiles and treatment response. Adicciones, 29, 125-133. doi:10.20882/adicciones.890.

McDonald, R. P. (1999). Test theory: a unified treatment. Mahwah, NJ: Lawrence Erlbaum. 
McGrath, D. S., Stewart, S. H., Klein, R. M. y Barrett, S. P. (2010). Self-generated motives for gambling in two population-based samples of gamblers. International Gambling Studies, 10, 117-138. doi:10.1080/14459795.2010.499915.

Moragas, L., Granero, R., Stinchfield, R., Fernández-Aranda, F., Fröberg, F., Aymamí, N., ... Jiménez-Murcia, S. (2015). Comparative analysis of distinct phenotypes in gambling disorder based on gambling preferences. $B M C$ Psychiatry, 15, 1-11. doi:10.1186/s12888-015-0459-0.

Muñiz, J., Elosua, P. y Hambleton, R. K. (2013). Directrices para la traducción y adaptación de los tests: segunda edición. Psicothema, 25, 151-157. doi:10.7334/psicothema2013.24.

Myrseth, H. y Notelaers, G. (2017). Is the Gambling Motives Questionnaire really three-dimensional? A proposition of a four-dimensional Gambling Motives Questionnaire-revised. Addictive Behaviors, 65, 68-73. doi:10.1016/j. addbeh.2016.10.002.

Navas, J. F., Billieux, J., Perandrés-Gómez, A., López-Torrecillas, F., Cándido, A. y Perales, J. C. (2017). Impulsivity traits and gambling cognitions associated with gambling preferences and clinical status. International Gambling Studies, 17, 1-23. doi:10.1080/14459795.2016.1275739.

Odlaug, B. L., Marsh, P. J., Won Kim, S. y Grant, J. E. (2011). Strategic vs. nonstrategic gambling: characteristics of pathological gamblers based on gambling preference. Annals of Clinical Psychiatry, 23, 105-112.

Potenza, M. N., Steinberg, M. A., McLaughlin, S. D., Wu, R., Rounsaville, B. J. y O'Malley, S. S. (2001). Gender-related differences in the characteristics of problem gamblers using a gambling helpline. American Journal of Psychiatry, 158, 1500-1505. doi:10.1176/appi.ajp.158.9.1500.

Stewart, S. H. y Zack, M. (2008). Development and psychometric evaluation of a three-dimensional Gambling Motives Questionnaire. Addiction, 103, 1110-1117. doi:10.1111/j.1360-0443.2008.02235.x.

Stewart, S. H., Zack, M., Collins, P. y Klein, R. M. (2008). Subtyping pathological gamblers on the basis of affective motivations for gambling: Relations to gambling problems, drinking problems, and affective motivations for drinking. Psychology of Addictive Behaviors, 22, 257-268. doi:10.1037/0893-164X.22.2.257.

St-Pierre, R. A., Walker, D. M., Derevensky, J. y Gupta, R. (2014). How availability and accessibility of gambling venues influence problem gambling: A review of the literature. Gaming Law Review and Economics, 18, 150-172. doi:10.1089/glre.2014.1824.

Tabachnick, B. G. y Fidell, L. S. (2007). Using Multivariate Statistics (5th ed.). New York: Allyn and Bacon.

Wardle, H., Moody, A., Spence, S., Orford, J., Volberg, R., Jotangia, D., ... Dobbie, F. (2011). British gambling prevalence survey 2010. London: National Centre for Social Research.

Winters, K. C., Stinchfield, R. D. y Fulkerson, J. (1993). Toward the development of an adolescent gambling problem severity scale. Journal of Gambling Studies, 9, 6384. doi:10.1007/BF01019925.

Zuckerman, M. (1994). Behavioral expressions and biosocial bases of sensation seeking. Cambridge, UK: Cambridge University Press.

Apéndice. Cuestionario de Motivos de Juego

A continuación, aparecerá una serie de motivos por los cuales es posible que juegues. Por favor, señala con qué frecuencia juegas por cada uno de los siguientes motivos.

\begin{tabular}{|c|c|c|c|c|}
\hline ¿Cón qué frecuencia juegas... & $\begin{array}{c}\text { Nunca } \\
\text { o casi nunca }\end{array}$ & Algunas veces & A menudo & $\begin{array}{c}\text { Casi siempre } \\
\text { o siempre }\end{array}$ \\
\hline porque te gusta lo que sientes? & 1 & 2 & 3 & 4 \\
\hline porque te hace sentir bien? & 1 & 2 & 3 & 4 \\
\hline porque es divertido? & 1 & 2 & 3 & 4 \\
\hline para olvidar las preocupaciones? & 1 & 2 & 3 & 4 \\
\hline porque te ayuda cuando te sientes nervioso o deprimido? & 1 & 2 & 3 & 4 \\
\hline para levantarte el ánimo cuando te sientes mal? & 1 & 2 & 3 & 4 \\
\hline porque te sientes más seguro de ti mismo? & 1 & 2 & 3 & 4 \\
\hline para relajarte? & 1 & 2 & 3 & 4 \\
\hline porque es lo que la mayoría de tus amigos hace cuando se juntan? & 1 & 2 & 3 & 4 \\
\hline porque esto hace que una reunión de amigos sea más agradable? & 1 & 2 & 3 & 4 \\
\hline como una manera de celebración? & 1 & 2 & 3 & 4 \\
\hline porque es algo que haces en ocasiones especiales? & 1 & 2 & 3 & 4 \\
\hline
\end{tabular}

